\title{
Utility of Platelet Indices in Diagnosing The Underlying Cause of Thrombocytopenia Due To Accelerated Platelet Destruction
}

\author{
Sushma Yalavarthi*, Nirvana Rasaily Halder and Aparna Pagidikalava \\ Department of Pathology, Mamata Medical college, Khammam, Telangana, India
}

\section{ABSTRACT}

Background: Thrombocytopenia is a common clinical manifestation of many diseases and has numerous causes, including decreased bone marrow production, increased spleen sequestration, and accelerated destruction of platelets. Among the various aetiologies, Accelerated platelet destruction is the most common cause. Distinction between the various aetiological categories of thrombocytopenia is usually made by invasive bone marrow examination. Some studies stated that Platelet indices were altered in various causes of thrombocytopenia and helpful in discriminating the causes. we aim, to know the role of platelet indices in determining the underlying cause of thrombocytopenia due to platelet destruction.

Methods: 30 Healthy controls and 145 patients (study group) with thrombocytopenia (platelet count $<150 x 109$ ) were included in the study. Thrombocytopenia due to decreased bonemarrow production and artefactual thrombocytopenia were excluded. The study group was divided into 3 groups. Group 1 includes immune thrombocytopenia, Group 2 having thrombocytopenia of non immunological causes and Group 3 includes miscellaneous causes. Clinical features, Platelet counts and platelet indices were studied and statistical analysis was performed.

Result: In all the groups of thrombocytopenia due to accelerated platelet destruction, MPV and P-LCR were significantly higher; P-LCC was significantly lower than healthy controls. There was no significant variation of PDW in NonImmune thrombocytopenia and miscellaneous groups, but PDW was significantly lower in immune thrombocytopenia than healthy controls.

Conclusion: Platelet indices should be considered in the diagnosis of thrombocytopenia due to accelerated destruction of any cause. Among the platelet indices, PDW can be useful for a positive diagnosis of immunological thrombocytopenia.

Keywords: Platelet Indices, Thrombocytopenia Due To Accelerated Destruction, Immune, Nonimmune, Miscellaneous.

\section{Introduction}

Among the various pathogenetic mechanisms of thrombocytopenia, the most common mechanism is Accelerated destruction. When the platelet destruction exceeds the compensatory increase in platelet production, thrombocytopenia develops. Platelet destruction may result from both intracorpuscular and extracorpuscular abnormalities. Intracorpuscular defects are rare. Platelet destruction most often is the result of extracorpuscular factors like immunological, non immunological and miscellaneous causes. ${ }^{[1]}$ Platelet counts below normal values define thrombocytopenia but do not reveal the underlying pathomechanism. The gold standard method for discriminating these causes is bone marrow examination. No consensus is reached regarding the necessity of a bone marrow examination in the evaluation of thrombocytopenia due to accelerated platelet destruction. Due to its invasiveness and being painful for the patients, this procedure is not recommended as first line diagnosis. ${ }^{[2]}$ Advances in automated blood cell analyzers have made it possible to measure various biomarkers of platelet activation which may provide some important information in diagnosing the underlying condition. ${ }^{2}$ The present study was conducted to demonstrate the discriminating potential of the platelet indices between Immune, Nonimmune and Miscellaneous causes of thrombocytopenia.

\section{Materials and Methods}

The present study included 30 Healthy controls and 145 patients (study group) with thrombocytopenia (platelet count $\left.<150 \times 10^{9}\right)$. Thrombocytopenia due to decreased bonemarrow production and artifactual thrombocytopenia were excluded. The study group was divided into 3 groups. Group 1 includes Thrombocytopenia due to immunological causes, Group 2 having thrombocytopenia of non immunological causes and Group 3 includes miscellaneous causes. The immune thrombocytopenia group included (group I) Autoimmune thrombocytopenia due to ITP and secondary causes like drugs, SLE, infections (HIV, Hepatitis B and C, Tuberculosis, Cytomegalovirus, Infectious Mononucleosis, Varicella or Zoster), lymphoproliferative disorders and thyroid disorders. The Non immunological causes included for Group II are Acute Pancreatitis, DIC, Hemolytic uremic syndrome, septicemia, pneumonia, septic shock, burns, 
snake bite, infections, hypothermia, platelet agglutinating drugs, coronary artery bypass, stenosed or artificial heart valves, cancers. Miscellaneous causes of group III included thrombocytopenias due to bacterial and viral infections such as Dengue, Malaria, Typhoid and other gram negative or gram positive infections. Assessment of complete blood count, MPV, PDW, P- LCR and P-LCC was done on Beckman Coulter. All whole-blood counts were assayed within $2 \mathrm{~h}$ of sample collection. Microscopic examination of a peripheral blood film stained with Leishman stain was done wherever necessary.

Statistics: Patient data were tabulated and processed using SPSS (17.0; SPSS Inc., Chicago, Illinois, USA) for Windows XP. Quantitative variables were expressed as mean, SD, and range and analyzed using Student's unpaired $t$-test. Qualitative data were expressed as frequency and percentage and were analyzed using the $\chi^{2}$-test. $P$-value less than 0.05 were considered statistically significant.

\section{Result}

A total of 145 thrombocytopenic patients and 30 healthy controls were reviewed in this study. These thrombocytopenic patients were divided into three categories based on the underlying cause. Group lincludes 7 patients with thrombocytopenia due to immunological causes, Group 2 includes 14 patients with nonimmunological thrombocytopenia, Group3 consists of 124 patients having thrombocytopenia due to miscellaneous causes.

The age of the patients ranged from 2 days to 80 years. The oldest case (78 years) was having nonimmune thrombocytopenia due to chronic kidney disease and pneumonia and the youngest (2 days) was diagnosed as neonatal thrombocytopenia due to respiratory distress syndrome. Maximum numbers of thrombocytopenia cases were reported in second to third decades. Healthy controls were also considered in third to fifth decades. Female patients comprised 68 and male patients 77 of total cases.
The aetiological distribution revealed, most of the thrombocytopenia cases $(85.5 \%)$ were due to miscellaneous causes. The different causes of thrombocytopenia were analyzed for the three patient groups along with the respective platelet count and platelet indices. The main causes of thrombocytopenia in immune thrombocytopenia are ITP, Viral Hepatitis, Tuberculosis and Thyroid disease. Nonimmune thrombocytopenia is due to Sepsis, septic shock, pneumonia, effusions, meningitis, drugs and toxins, snake bite and Acute Pancreatitis. Most common aetiology in the present study is Infections. Infections contribute 124 cases $(85.5 \%)$. Miscellaneous causes for thrombocytopenia in the present study are Infections like Malaria, Dengue, Typhoid and viral, Hypersplenism, chronic liver disease, chronic kidney disease or Diabetes Mellitus. (Table 1)

The platelet count and the platelet indices were compared between the three groups and healthy controls. In healthy controls, MPV Values are 9.74 \pm 1.106 , PDW is $12.16 \pm 2.015$, P-LCR is $33.88 \pm 11.88$ and P-LCC is $74.53 \pm 20.59$. In all the three groups of individuals; MPV and P-LCR are significantly higher than healthy controls with the mean of $17.43 \pm 3.69$ and $36.86 \pm 27.39$ (Group1), $17.57 \pm 4.89$ and $37.57 \pm 15.45$ (Group 2), 15.09 \pm 3.51 and $33.88 \pm 11.88$ (Group 3) respectively. P-LCC values are lower than controls with the mean of $17.10 \pm 15.74$ (Group 1), 20.36 \pm 6.640 (Group 2) and 18.71 \pm 10.18 (Group 3). But PDW is significantly decreased $(8.86 \pm 6.122)$ in Immune Thrombocytopenia (ITP) than the other two groups. In other two groups no significant decrease noted-

Platelet count, MPV and P-LCR were significantly higher $(P<0.0001)$ in all the three groups as compared to healthy controls. P-LCC is significantly lower than healthy controls. There is no significant variation of PDW in NonImmune thrombocytopenia and miscellaneous groups, but PDW is significantly lower in immune thrombocytopenia than healthy controls. (Tables 2-4)

Table 1: Distribution of Thrombocytopenia cases based on underlying pathogenetic mechanism .

\begin{tabular}{|l|l|l|l|l|}
\hline & $\begin{array}{l}\text { Group 1 } \\
\text { (Immunological) }\end{array}$ & $\begin{array}{l}\text { Group 2 (Non } \\
\text { Immunological) }\end{array}$ & $\begin{array}{l}\text { Group 3 } \\
\text { (Miscellaneous) }\end{array}$ & Total \\
\hline Aetiology & $\begin{array}{l}\text { ITP, Viral Hepatitis, } \\
\text { Tuberculosis, Thyroid } \\
\text { disease }\end{array}$ & $\begin{array}{l}\text { Sepsis, septic shock, } \\
\text { pneumonia, effusions, } \\
\text { meningitis, Acute } \\
\text { pancreatitis, drugs } \\
\text { and toxins, snake bite, } \\
\text { Acute Pancreatitis }\end{array}$ & $\begin{array}{l}\text { Infections like Malaria, } \\
\text { Dengue, and Typhoid, } \\
\text { viral. Hypersplenism, } \\
\text { chronic liver disease, } \\
\text { chronic kidney disease } \\
\text { or Diabetes Mellitus }\end{array}$ & \\
\hline Number & 7 & 14 & 124 & 145 \\
\hline$(\%)$ & 4.8 & 9.7 & 85.5 & 100 \\
\hline
\end{tabular}


Table 2: comparison of platelet count and platelet indices between Group 1 and control group.

\begin{tabular}{|l|c|c|c|}
\hline Platelet count and indices & Group 1 & Control group & p-value \\
\hline Platelet count (mean \pm SD) & $36.71 \pm 13.66$ & $303.53 \pm 22.18$ & $<0.0001$ \\
\hline MPV(mean \pm SD) & $17.43 \pm 3.69$ & $9.74 \pm 1.106$ & $<0.0001$ \\
\hline PDW $($ mean \pm SD) & $8.86 \pm 6.122$ & $12.16 \pm 2.015$ & $=0.0167$ \\
\hline P-LCR $($ mean \pm SD) & $36.86 \pm 27.39$ & $24.87 \pm 7.196$ & $=0.0360$ \\
\hline P-LCC $($ mean \pm SD) & $17.10 \pm 15.74$ & $74.53 \pm 20.591$ & $<0.0001$ \\
\hline
\end{tabular}

Table 3: comparison of platelet count and platelet indices between Group 2 and control group.

\begin{tabular}{|c|c|c|c|}
\hline Platelet count and indices & Group 2 & Control group & $p$-value \\
\hline Platelet count (mean \pm SD) & $46.71 \pm 19.37$ & $303.53 \pm 22.18$ & $<0.0001$ \\
\hline $\mathrm{MPV}($ mean $\pm \mathrm{SD})$ & $17.57 \pm 4.89$ & $9.74 \pm 1.106$ & $<0.0001$ \\
\hline PDW(mean \pm SD) & $11.14 \pm 4.44$ & $12.16 \pm 2.015$ & $=0.2966$ \\
\hline P-LCR(mean \pm SD) & $37.57 \pm 15.45$ & $24.87 \pm 7.196$ & $=0.0005$ \\
\hline P-LCC(mean \pm SD) & $20.36 \pm 6.640$ & $74.53 \pm 20.591$ & $<0.0001$ \\
\hline
\end{tabular}

Table 4: comparison of platelet count and platelet indices between Group 3 and control group.

\begin{tabular}{|l|c|c|c|}
\hline Platelet count and indices & Group 3 & Control group & p-value \\
\hline Platelet count (mean \pm SD) & $54.74 \pm 29.17$ & $303.53 \pm 22.18$ & $<0.0001$ \\
\hline MPV(mean \pm SD) & $15.09 \pm 3.506$ & $9.74 \pm 1.106$ & $<0.0001$ \\
\hline PDW $($ mean \pm SD) & $11.86 \pm 4.834$ & $12.16 \pm 2.015$ & $=0.7401$ \\
\hline P-LCR(mean \pm SD) & $33.88 \pm 11.88$ & $24.87 \pm 7.196$ & $=0.0002$ \\
\hline P-LCC $($ mean \pm SD) & $18.71 \pm 10.18$ & $74.53 \pm 20.591$ & $<0.0001$ \\
\hline
\end{tabular}

\section{Discussion}

Platelet indices are biomarkers of platelet activation. Platelet indices (PI) — Mean platelet volume (MPV) and platelet distribution width (PDW), Platelet - Large cell ratio (P-LCR), Platelet- Large cell coefficient (P-LCC) - are a group of derived platelet parameters obtained as a part of the automated complete blood count. Emerging evidence suggests that PIs may allow extensive clinical investigations focusing on the diagnostic and prognostic values in a variety of settings without bringing extra costs. ${ }^{[3]}$

The volume of platelets in the bloodstream is heterogeneous, and their structures and metabolic functions differ. Typically, the average mean cell volume is $7.2-11.7 \mathrm{fL}$ in healthy subjects. ${ }^{[4,5]} \mathrm{MPV}$ is determined in the progenitor cell, the bone marrow megakaryocyte. When platelet production is decreased, young platelets become bigger and more active, and MPV levels increase. Increased MPV indicates increased platelet diameter, which can be used as a marker of production rate and platelet activation. During activation, platelets' shapes change from biconcave discs to spherical, and a pronounced pseudopod formation occurs that leads to MPV increase during platelet activation. MPV changes are complex, and are not only related to the PLT count, but also related to the method of laboratory analysis used. ${ }^{[6,7]}$ In a study by Akarsu et al, a MPV $>9.5$ fl was considered above normal range, ${ }^{[8]}$ and in another study, MPV elevation was defined $>10.4 \mathrm{fl}$, both of which were commonly found in our study. The normal range of MPV from our laboratory was $9-17 \mathrm{fl}^{[3]}$

Our study showed that Platelet count, MPV and P-LCR were significantly higher $(P<0.0001)$ in all the three groups as compared to healthy controls. P-LCC is significantly lower than healthy controls. There is no significant variation of PDW in NonImmune thrombocytopenia and miscellaneous groups, but PDW is significantly lower in immune thrombocytopenia. Borkataky et al. ${ }^{[9]}$ found no significant difference in the MPV between the destructive thrombocytopenia groups and the control group. Similarly, previous work by researchers such as Kaito et al. ${ }^{[10]}$ , Ntaios et al. ${ }^{[11]}$ and Shah et al. ${ }^{[12]}$ reported that MPV was higher in ITP patients, which reflected an increase in the production rate, and they established cutoff values ranging from greater than $9 \mathrm{fl}$ to greater than $11 \mathrm{fl}$. Although Numbenjapon et al reported that MPV could be usedindistinguishinghyperdestructive fromhypoproductive thrombocytopenia; they proposed a cutoff value of $7.9 \mathrm{fl}$, which is lower than the previously reported cutoff values. ${ }^{[13]}$ PDW is an indicator of volume variability in platelets size and is increased in the presence of platelet anisocytosis 
.The PDW reported varies markedly, with reference intervals ranging from 8.3 to $56.6 \% .^{[4,6-7]}$. PDW directly measures variability in platelet size, changes with platelet activation, and reflects the heterogeneity in platelet morphology. ${ }^{[, 7]}$ Under physiological conditions, there is a direct relationship between MPV and PDW; both usually change in the same direction. ${ }^{[5]}$ Meanwhile, there are conflicting reports in the literature about the relationship between platelet volume and numbers, which suggests that they are affected by different mechanisms. ${ }^{[3]}$ In our study also, we observed that MPV and PDW changed in same direction.

Platelet larger cell ratio (P-LCR) is an indicator of circulating larger platelets $(>12 \mathrm{fL})$, which is presented as percentage. The normal percentage range is $15-35 \%$. It has also been used to monitor platelet activity. Platelet large cell ratio, PLCR, is often correlated to MPV but is more sensitive to changes in platelet size. Babu et al has shown its level is inversely related to the platelet counts and directly related to MPV and PDW, and is an aid for the differentiation of thrombocytopenia. ${ }^{[14]}$

Platelet large cell Coefficient (P-LCC) means number of platelets larger than $12 \mathrm{fl}$ and smaller than $30 \mathrm{fl}$. P-LCR is calculated in automated blood analyzers using this formula- P-LCC/ PLT. Another explanation for the differences in the cutoff values of Platelet indices in different studies can be the difference in the type of the hematological analyzer used. Moreover, they do not count large or giant platelets because they cannot be differentiated from red blood cells. Furthermore, many papers in the literature have shown that MPV is dependent on a number of variables, including the time of analysis after venipuncture, the anticoagulant used, the specimen storage temperature, and counter technologies. In the present study, the P-LCR was significantly higher in in all the three groups of thrombocytopenia when compared with the control group. Similarly, Ntaios et al. ${ }^{[1]]}$ and Kaito et al. ${ }^{[10]}$ reported nearly similar cutoff value of greater than $30 \%$, with diagnostic sensitivities of 90.4 and $91.4 \%$, respectively. In addition, Babu and Basu ${ }^{[15]}$ and Borkataky et al. ${ }^{[9]}$ reported that the P-LCR was increased in destructive thrombocytopenia patients compared with healthy controls and they concluded that the P-LCR can be a good aid in the differential diagnosis of conditions associated with abnormal platelet counts. With regard to PDW, there was no significant difference between the two patient groups or between the patient groups and the control group in our study. In contrast, Shah et al. ${ }^{[12]}$ and Borkataky et al. ${ }^{[9]}$ found that the PDW was higher in ITP patients compared with acute myeloid leukemia patients and nonmegaloblastic hypoproliferative patients, respectively. In addition, Kaito et al. [10] suggested a cutoff value of greater than $17 \mathrm{fl}$ for PDW to distinguish ITP from hypoproductive thrombocytopenia, with $71.8 \%$ diagnostic sensitivity and $95 \%$ specificity. Similarly, Ntaios et al. ${ }^{[1]}$ suggested a cutoff value between 15 and $17 \mathrm{fl}$, with 100\% sensitivity, specificity, positive predictive value, and negative predictive value. In our study, PDW values are significantly lower in Immune thrombocytopenia (ITP) than Nonimmune and miscellaneousgroupsinwhomthereisnosignificantvariation. In the future, improved research designs and standardized measurements for platelet indices may significantly increase the diagnostic predictive power of platelet indices in the differential diagnosis of thrombocytopenia.

\section{Conclusion}

Platelet indices should be considered in the diagnosis of thrombocytopenia due to accelerated destruction of any cause. No significant variation in the MPV, P-LCR, P-LCC in all the three groups. The PDW can be useful for a positive diagnosis of immunological thrombocytopenia (ITP).

\section{Reference}

1. Levine SP. Thrombocytopenia: Pathophysiology and classification. In: Greer JP, Forester J, Leukens JN, Rodgers GM, Paraskevas F, Glader B editors. Wintrobe's Clinical Hematology, 11th ed. Philadelphia: Lippincott Williams\& Wilkins; 2004.1529.

2. Mikias N, Aster T, Amha GM, et al. Diagnostic predictive value of platelet indices for discriminating hypo productive versus immune thrombocytopenia purpura in patients attending a tertiary care teaching hospital in Addis Ababa, Ethiopia. BMC Hematol. 2016;16:18.

3. Yasemin UB, Murat P, Huysa K, et al. .The use of platelet indices, plateletcrit, mean platelet volume and platelet distribution width in emergency non-traumatic abdominal surgery: a systematic review. Biochem Med . 2016;26:178193.

4. Demirin H, Ozhan H, Ucgun T, Celer A, Bulur S, Cil H, et al. Normal range of mean platelet volume in healthy subjects: insight from a large epidemiologic study. Thromb Res. 2011;128: 358-60.

5. Wiwanitkit V. Plateletcrit, mean platelet volume, platelet distribution width: its expected values and correlation with parallel red blood cell parameters. Clin Appl Thromb Hemost. 2004;10:175-8.

6. Maluf CB, Barreto SM, Vidigal PG. Standardization and reference intervals of platelet volume indices: Insight from the Brazilian longitudinal study of adult health (ELSABRASIL). Platelets. 2015;26:413-20.

7. Vagdatli E, Gounari E, Lazaridou E, Katsibourlia E, Tsikopoulou F, Labrianou I. Platelet distribution width: a simple, practical and specific marker of activation of coagulation. Hippokratia. 2010;14:28-32. 
8. Akarsu S, Taskin E, Kilic M, Ozdiller S, Gurgoze MK, et al. The effects of different infectious organisms on platelet counts and platelet indices in neonates with sepsis: is there an organism-specific response? J Trop Pediatr.2005;51:388-91.

9. Borkataky S, Jain R, Gupta R, Singh S, Krishnan G, Gupta $\mathrm{K}$, et al. Role of platelet volume indices in the differential diagnosis of thrombocytopenia: a simple and inexpensive method. Hematology 2009;14:182-186.

10. Kaito K, Otsubo H, Usui N, Yoshida M, Tanno J, Kurihara E, et al. Platelet size deviation width, platelet large cell ratio, and mean platelet volume have sufficient sensitivity and specificity in the diagnosis of immune thrombocytopenia. Br J Haematol 2005;128:698-702.

11. Ntaios G, Papadopoulos A, Chatzinikolaou A, Saouli Z, Karalazou P, Kaiafa G, et al. Increased values of mean platelet volume and platelet size deviation width may provide a safe positive diagnosis of idiopathic thrombocytopenic purpura. Acta Haematol 2008;119:173-177.

12. Shah AR, Chaudhari SN, Shah $\mathrm{MH}$, et al. Role of platelet parameters in diagnosing various clinical conditions. Natl J Med Res 2013;3:162-165.
13. Elsewefy DA, Farweez BA, Ibrahim RR. Platelet indices: consideration in thrombocytopenia.Egyptian Journal of Hamatology 2014;39:134-138.

14. Osselaer JC, Jamart J, Scheiff JM. Platelet distribution width for differential diagnosis of thrombocytosis. Clin Chem. 1997;43:1072-6.

15. Babu E, Basu D. Platelet large cell ratio in the differential diagnosis of abnormal platelet counts. Indian $\mathrm{J}$ Pathol Microbiol 2004;47:202-205.

16. Sachdev R, Tiwari AK, Goel S, Raina V, Sethi M. Establishing biological reference intervals for novel platelet parameters (immature platelet fraction, high immature platelet fraction, platelet distribution width, platelet large cell ratio, platelet-X, plateletcrit, and platelet distribution width) and their correlations among each other. Indian J Pathol Microbiol. 2014;57:231-5.

17. Provan D, Stasi R, Newland AC, Blanchette VS, BoltonMaggs P, Bussel JB, et al. International consensus report on the investigation and management of primary immune thrombocytopenia. Blood 2010;115:168-186.

*Corresponding author:

Sushma Yalavarthi, Professor, Department of Pathology, Mamata Medical college, Khammam, Telangana, India

Phone: +91 09848676441

Email: yalavarthisushma@yahoo.co.in

Financial or other Competing Interests: None. 